\title{
Sustainable and Selective Separation of PVC and ABS from a WEEE Plastic Mixture using Microwave and/or Mild-heat Treatment with Froth Flotation \\ Nguyen Thi Thanh Truc, Byeong-Kyu Lee* \\ Department of Civil and Environmental Engineering, University of Ulsan, Daehakro 93, Namgu, Ulsan 680-749, Republic of Korea
}

Corresponding Author: *Byeong-Kyu Lee, Professor, Tel: 82-52-259-2864, Fax: 82-52-259-2629, E-mail: $\underline{\text { bklee@ulsan.ac.kr }}$

\section{Supporting Information}

Supporting information includes 8 pages with 2 figures and 4 tables and references in the tables.

Fig. S1. Surface modification of plastics by microwave/ heat treatment and froth flotation experiment

Fig. S2. WEEE and WEEE plastics

Table S1. WEEE plastic composition (\%) of this study and previous reports

Table S2. The contact angles of plastic before and after the treatments and the disparity in CAs between PVC and the other plastics after each of the treatment

Table S3. Root mean square (RMS) value (nm) of plastics before and after PAC coating with microwave treatment (PAC@MW) and PAC coating with mild-heat treatment (PAC@MH).

Table S4. ABS and PVC separation from plastic mixture in some studies 


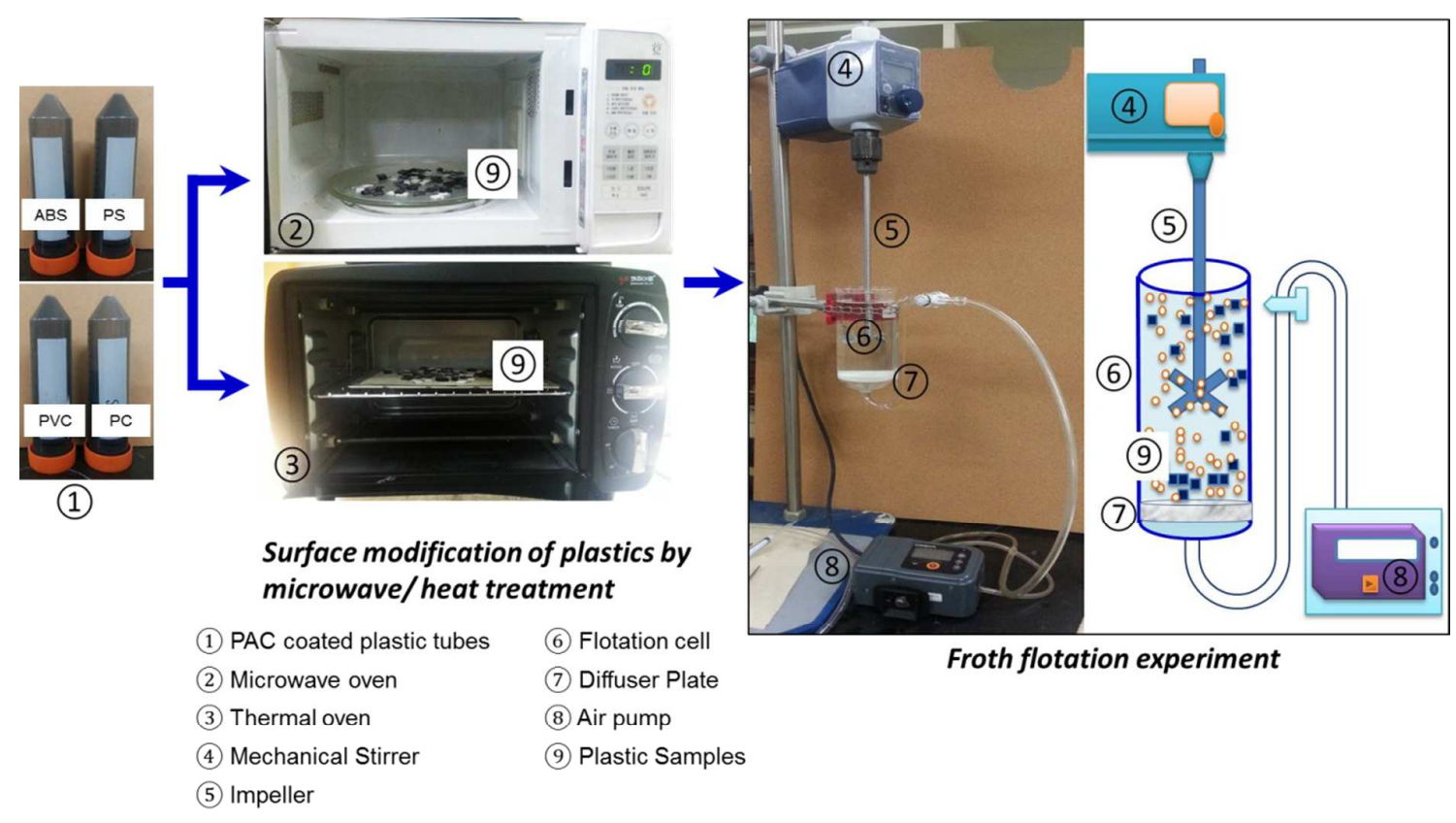

Fig. S1. Surface modification of plastics by microwave/ heat treatment and froth flotation experiment 


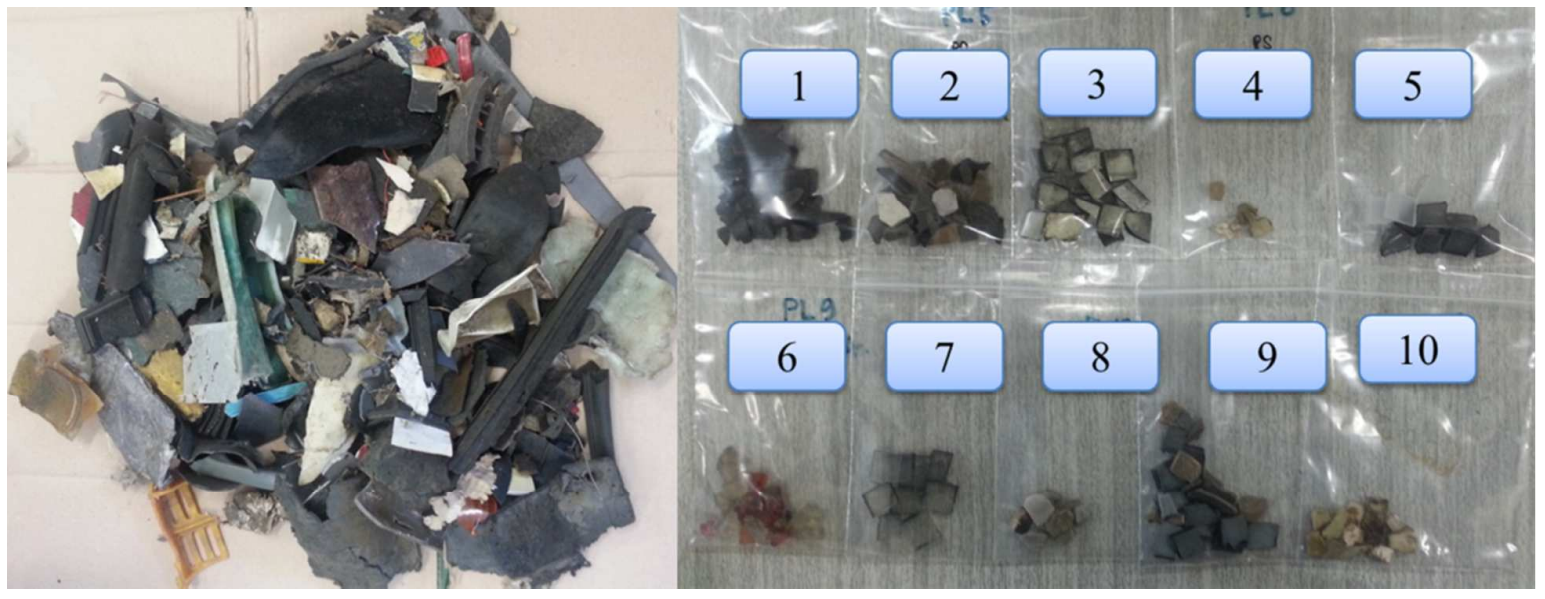

Fig. S2. WEEE and WEEE plastics 
Table S1. WEEE plastic composition (\%) of this study and previous reports

\begin{tabular}{ccccc}
\hline \hline Type of plastic & This study & Martinho t al. $^{l}$ & Yang et al. $^{2}$ & Maris et al. $^{3}$ \\
\hline PP and PE & $17.77 \pm 2.10$ & $1-20$ & 8 & 22 \\
PET & $2.18 \pm 0.06$ & & & \\
ABS & $41.35 \pm 1.60$ & $6-69$ & 30 & 29 \\
HIPS & $2.74 \pm 1.50$ & $0-21$ & 25 & 26 \\
PS & $10.37 \pm 0.40$ & $4-76$ & 3 & 3 \\
PMMA & $2.46 \pm 0.20$ & & & 8 \\
PC & $6.52 \pm 0.60$ & $1-23$ & 19 & 1 \\
PA & $3.30 \pm 0.04$ & & 3 & \\
PVC & $9.87 \pm 0.20$ & & 3 & 11 \\
POM & $1.00 \pm 0.60$ & & 9 & \\
Other & $2.45 \pm 0.45$ & & & \\
\hline \hline
\end{tabular}


Table S2. The contact angles of plastic before and after the treatments and the disparity in CAs between PVC and the other plastics after each of the treatment

\begin{tabular}{|c|c|c|c|c|c|c|c|c|c|}
\hline \multirow{2}{*}{ Plastic } & \multirow{2}{*}{ Before } & \multicolumn{2}{|c|}{ MW } & \multicolumn{2}{|c|}{ PAC@MW } & \multicolumn{2}{|c|}{ MH } & \multicolumn{2}{|c|}{ PAC@MH } \\
\hline & & CAs* & $\triangle \mathrm{PVC}^{* *}$ & $\mathrm{CA}$ & $\triangle \mathrm{PVC}$ & $\mathrm{CA}$ & $\triangle \mathrm{PVC}$ & $\mathrm{CA}$ & $\triangle \mathrm{PVC}$ \\
\hline PC & 87.7 & 84.7 & 4 & 89.5 & 19.6 & 85.6 & 4.9 & 76.0 & 22.3 \\
\hline PVC & 86.8 & 88.7 & 0 & 69.9 & 0 & 90.5 & 0 & 98.3 & 0 \\
\hline ABS & 78.8 & 81.4 & 7.3 & 81.0 & 11.1 & 82.1 & 8.4 & 75.4 & 22.9 \\
\hline PS & 80.5 & 87.4 & 1.3 & 90.2 & 20.3 & 70.8 & 19.7 & 67.2 & 31.1 \\
\hline
\end{tabular}

*CAs: the contact angles of the plastics

** $\triangle P V C$ : the disparity in contact angle between PVC and the PC, ABS and PS. 
Table S3. Root mean square (RMS) value (nm) of plastics before and after PAC coating with microwave treatment (PAC@MW) and PAC coating with mild-heat treatment (PAC@MH).

\begin{tabular}{cccc}
\hline Plastics & Before & PAC@MW & PAC@MH \\
\hline PC & $3.0 \pm 0.10$ & $10.8 \pm 0.01$ & $3.2 \pm 0.03$ \\
PVC & $4.3 \pm 0.21$ & $7.5 \pm 0.03$ & $18.8 \pm 0.12$ \\
ABS & $8.3 \pm 0.26$ & $12.2 \pm 0.06$ & $30.5 \pm 0.31$ \\
PS & $9.6 \pm 0.12$ & $13.3 \pm 0.21$ & $33.4 \pm 0.17$ \\
\hline \hline
\end{tabular}


Table S4. ABS and PVC separation from plastic mixture in some studies

\begin{tabular}{|c|c|c|c|c|c|c|}
\hline \multirow[b]{2}{*}{ The method } & \multirow[b]{2}{*}{ Plastic mixture } & \multicolumn{2}{|c|}{$\overline{P V C}$} & \multicolumn{2}{|c|}{$\overline{\mathrm{ABBS}}$} & \multirow[b]{2}{*}{ Sources } \\
\hline & & $\begin{array}{c}\text { Recovery } \\
(\%)\end{array}$ & $\begin{array}{c}\text { Purity } \\
(\%)\end{array}$ & $\begin{array}{c}\text { Recovery } \\
(\%)\end{array}$ & $\begin{array}{l}\text { Purity } \\
(\%)\end{array}$ & \\
\hline $\begin{array}{c}\text { Triboelectrostatic } \\
\text { separation }\end{array}$ & $\begin{array}{c}\text { ABS, PS and } \\
\text { PP }\end{array}$ & & & $>73.0$ & 92.1 & ${\text { Dodbiba } \text { et } \text { al. }{ }^{4}}^{4}$ \\
\hline $\begin{array}{l}\text { Dissolved air } \\
\text { flotation }\end{array}$ & ABS and PS & & & 89.38 & 97.24 & Hui et al. ${ }^{5}$ \\
\hline Ammonia $+\mathrm{FF}^{*}$ & $\mathrm{PC}$ and $\mathrm{ABS}$ & & & 95.35 & 99.23 & Wang et al. ${ }^{6}$ \\
\hline $\begin{array}{l}\text { Boiling treatment }+ \\
\text { FF }\end{array}$ & ABS and PS & & & 95.81 & 95.80 & Wang et al. ${ }^{7}$ \\
\hline Tannic acid + FF & PET and PVC & 91.89 & 96.34 & & & Abbasi et al. ${ }^{8}$ \\
\hline $\begin{array}{l}\text { Gelatin and lignin } \\
\text { alkali + FF }\end{array}$ & PET and PVC & & 98.9 & & & Yenial et al. ${ }^{9}$ \\
\hline $\mathrm{CaLS}+\mathrm{FF}$ & PET and PVC & 100 & 100 & & & Saisinchai ${ }^{10}$ \\
\hline Ozonation + FF & $\begin{array}{l}\text { PVC, PET, PC, } \\
\text { and PMMA }\end{array}$ & 90 & 100 & & & Reddy et al. ${ }^{11}$ \\
\hline $\begin{array}{l}\mathrm{KMnO}_{4} \text {, ultrasonic } \\
\quad+\mathrm{FF}\end{array}$ & PET and PVC & 99.73 & 99.30 & & & Wang et al. ${ }^{12}$ \\
\hline $\begin{array}{l}\text { Nanometallic } \\
\qquad \mathrm{Ca} / \mathrm{CaO}+\mathrm{FF}\end{array}$ & E-waste plastics & 100 & 96.4 & & & Reddy et al. ${ }^{13}$ \\
\hline PAC@MW + FF & WEEE plastics & 100 & 100 & 0 & 0 & This study \\
\hline PAC@MH + FF & WEEE plastics & 100 & 96.8 & 100 & 100 & This study \\
\hline
\end{tabular}

*FF: froth flotation

\section{References}

1. Martinho, G.; Pires, A.; Saraiva, L.; Ribeiro, R., Composition of plastics from waste electrical and electronic equipment (WEEE) by direct sampling. Waste Manage. 2012, 32, 1213-1217.

2. Yang, X.; Sun, L.; Xiang, J.; Hu, S.; Su, S., Pyrolysis and dehalogenation of plastics from waste electrical and electronic equipment (WEEE): A review. Waste Manage. 2013, 33, 462-473.

3. Maris, E.; Botané, P.; Wavrer, P.; Froelich, D., Characterizing plastics originating from WEEE: A case study in France. Miner. Eng. 2015, 76, 28-37. 
4. Dodbiba, G.; Shibayama, A.; Miyazaki, T.; Fujita, T., Triboelectrostatic Separation of ABS, PS and PP Plastic Mixture. Mater. Trans. 2003, 44, 161 - 166.

5. Wang, H.; Chen, X.; Bai, Y.; Guo, C.; Zhang, L., Application of dissolved air flotation on separation of waste plastics ABS and PS. Waste Manage. 2012, 32, 1297-1305.

6. Wang, C. Q.; Wang, H.; Liu, Q.; Fu, J.; Liu, Y., Separation of polycarbonate and acrylonitrile-butadiene-styrene waste plastics by froth flotation combined with ammonia pretreatment. Waste Manage. 2014, 34, 2656-2661.

7. Wang, C. Q.; Wang, H.; Wu, B. X.; Liu, Q., Boiling treatment of ABS and PS plastics for flotation separation. Waste Manage. 2014, 34, 1206 -1210.

8. Abbasi, M.; Salarirad, M. M.; Ghasemi, I., Selective separation of PVC from PET/PVC mixture using floatation by tannic acid depressant. Iran. Polym. J. 2010, 19, 483-489.

9. Yenial, Ü.; Kangal, O.; Güney, A., Selective flotation of PVC using gelatin and lignin alkali. Waste Manage.Res. 2013, 31, 613-617.

10. Saisinchai, S., Separation of PVC from PET/PVC Mixtures Using Flotation by Calcium Lignosulfonate Depressant. Eng.J. 2014, 18, 45 - 53.

11. Reddy, M. S.; Okuda, T.; Kurose, K.; Tsai, T. Y.; Nakai, S.; Nishijima, W.; Okada, M., Surface ozonation of polyvinyl chloride for its separation from waste plastic mixture by froth fl oatation. J. Mater. Cycles Waste. 2010, 12, 326-331.

12. Wang, C.; Wang, H.; Fu, J.; Zhang, L.; Luo, C.; Liu, Y., Flotation separation of polyvinyl chloride and polyethylene terephthalate plastics combined with surface modification for recycling. Waste Manage. 2015, 45, 112-117.

13. Reddy, M. S.; Heo, J. H.; Park, M. H., Hybrid selective surface hydrophilization and froth flotation separation of hazardous chlorinated plastics from E-waste with novel nanoscale metallic calcium composite. J. Hazard. Mater. 2015, 306, 13-23. 